\title{
An efficient regularization method for a large scale ill-posed geothermal problem
}

Fredrik Berntsson, Lin Chen, Tao Xu and Dennis Wokiyi

The self-archived version of this journal article is available at Linköping University Electronic Press:

http:/ / urn.kb.se/ resolve?urn=urn:nbn:se:liu:diva-139052

N.B.: When citing this work, cite the original publication.

Berntsson, F., Chen, L., Xu, T., Wokiyi, D., (2017), An efficient regularization method for a large scale ill-posed geothermal problem, Computers \& Geosciences, 105, 1-9.

https:// dx.doi.org/ 10.1016/j.cageo.2017.04.010

Original publication available at:

https:/ / dx.doi.org/ 10.1016/j.cageo.2017.04.010

Copyright: Elsevier

http:// www.elsevier.com/

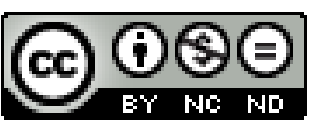




\title{
An efficient regularization method for a large scale ill-posed geothermal problem
}

\author{
Fredrik Berntsson, Chen Lin†, Tao Xu; and Dennis Wokiyi*
}

May 5, 2017

\begin{abstract}
The inverse geothermal problem consists of estimating the temperature distribution below the earth's surface using measurements on the surface. The problem is important since temperature governs a variety of geologic processes, including the generation of magmas and the deformation style of rocks. Since the thermal properties of rocks depend strongly on temperature the problem is non-linear.

The problem is formulated as an ill-posed operator equation, where the righthand side is the heat-flux at the surface level. Since the problem is ill-posed regularization is needed. In this study we demonstrate that Tikhonov regularization can be implemented efficiently for solving the operator equation. The algorithm is based on having a code for solving a well-posed problem related to the above mentioned operator. The algorithm is designed in such a way that it can deal with both $2 D$ and $3 D$ calculations.

Numerical results, for $2 D$ domains, show that the algorithm works well and the inverse problem can be solved accurately with a realistic noise level in the surface data.
\end{abstract}

*Email: fredrik.berntsson@liu.se. dennis.wokiyi@liu.se. Department of Mathematics, Linköping University, S-581 83, Linköping, Sweden.

†Email: chenlin@mail.iggcas.ac.cn. State Key Laboratory of Lithospheric Evolution, Institute of Geology and Geophysics, Chinese Academy of Sciences, Beijing 100029, China

${ }^{\ddagger}$ Email: xutao@mail.iggcas.ac.cn. State Key Laboratory of Lithospheric Evolution, Institute of Geology and Geophysics, Chinese Academy of Sciences, Beijing 100029, China, and also CAS Center for Excellence in Tibetan Plateau Earth Sciences, Beijing 100101, China. 


\section{Introduction}

24 Temperature controls many physical properties of rocks and governs a variety

\begin{abstract}
of geologic processes, including the generation of magmas and the deformation style of rocks $[1,3,20,26]$, see also [9, 10, 23]. Thus, knowledge of the thermal structure of the lithosphere is essential for understanding geophysical observations and dynamic processes in the deep interior. However, temperature cannot be directly measured beyond a few kilometers depth, in deep mines or drill holes $[12,21]$. To obtain the information about the temperature distribution in the lithosphere, the surface observations, e.g. surface heat flux, are extrapolated to the lithosphere base using a heat conduction model [5, 8]. Since the thermal conductivity of rocks are temperature and pressure dependent [11] the problem is non-linear. For the one-dimensional case an analytical solution can be found $[7,14]$ but for the two or three-dimensional case numerical modeling is required. Several previous studies investigated this type of problems using the least squares approach, see [32, 29], and also [19, 9], but these authors did not strictly treat the nonlinear aspect resulting from the temperature-dependent thermal properties of rocks.
\end{abstract}

The inverse geothermal problem can be formulated as a boundary value problem for the stationary heat equation in a domain, but where the temperature and heat-flux data are only known on a part of the boundary. This is often referred to as the Cauchy problem for the heat equation $[2,15]$, see also [4] and [5]. The problem is ill-posed and a regularization technique is needed to stabilize the computations, see [35] for a good overview of regularization techniques used in geophysics.

We reformulate the problem as a non-linear operator equation; where the operator maps the heat-flux at the lower boundary to the heat-flux at the surface level. The operator can thus be evaluated by solving a well-posed boundary value problem for the temperature distribution inside the domain under consideration. The inverse problem is solved by using Tikhonov regularization. In our implementation we solve the normal equations, that correspond to the minimization of the Tikhonov functional, using the conjugate gradient method. The bulk of the computational work in the algorithm consists of solving several linear systems of equations originating from a finite difference discretization of the underlying well-posed boundary value problem. We also show how to treat the non-linearity of the problem. An important feature of the algorithm is that it can be generalized to the case surface data is available on a $2 D$ area and the temperature distribution for a $3 D$ region below the surface is desired.

The outline of this paper is as follows: In Section 2 we introduce a well-posed boundary value problem for the heat-equation that allows us to reformulate the inverse geothermal problem as a non-linear operator equation. We also give the the details of our thermal model. In Section 3 we start by investigating 

the inverse geothermal problem for the case of a constant thermal conductivity and heat production. For this case we can use the singular value decomposition

68 Section 4 . We show how to implement Tikhonov regularization efficiently by 69 using the conjugate gradient method. Finally, we give numerical results in 70 Section 4.2 and present conclusions in Section 5.

\section{${ }_{71} 2$ The Direct Problem}

For geothermal calculations the goal is to estimate the temperature distribution in the lithosphere [9]. The model is two dimensional and confined to the domain

${ }_{74}(x, z) \in \Omega=\left(0, L_{x}\right) \times\left(0, L_{z}\right)$, where $x$ is the lateral coordinate, and $z$ is the 75 depth.

76 The boundary of the domain $\Omega$ consists of the surface where measurements 77 are available, the base where we specify the heat flux, and the sides where we 78 assume a zero flux. Thus the thermal model is the following: The temperature $79 \quad T(x, z) \in C^{2}(\Omega) \cap C^{1}(\bar{\Omega})$ satisfies,

$$
\begin{cases}\left(\kappa T_{x}\right)_{x}+\left(\kappa T_{z}\right)_{z}+A_{p}=0, & 0<x<L_{x}, \quad 0<z<L_{z}, \\ T_{x}(0, z)=T_{x}\left(L_{x}, z\right)=0, & 0<z<L_{z}, \\ T(x, 0)=T_{0}(x), & 0<x<L_{x}, \\ \kappa T_{z}\left(x, L_{z}\right)=Q_{m}(x), & 0<x<L_{x},\end{cases}
$$

so where $\kappa:=\kappa(x, z, T)$ is the thermal conductivity and $A_{p}:=A_{p}(x, z, T)$, or 81 more commonly $A_{p}:=A_{p}(x, z)$, is the heat production. The above problem is ${ }_{82}$ well-posed [13] and provided that we have access to the heat-flow $Q_{m}$ and the sз surface temperature $T_{0}$ we can compute the temperature distribution $T(x, z)$ 84 in the domain $\Omega$. This allows us to define a non-linear operator as follows: In 85 (2.1) we consider the surface temperature $T_{0}$ to be fixed. If, in addition, we 86 specify the heat-flux $Q_{m}$ then we have a well-posed problem that we can solve 87 for the temperature distribution $T(x, z)$ in the domain. Having obtained $T(x, z)$ ${ }_{88}$ we can compute the surface heat-flux $Q_{0}$. This means that we can introduce a 89 mapping from $Q_{m}$ to $Q_{0}$ that we denote by

$$
Q_{0}=\kappa T_{z}(x, 0)=\mathcal{K}\left(\kappa, A_{p}, T_{0}\right) Q_{m},
$$

90 In order to simplify the notation in the paper we also introduce a function

$$
\left[T, Q_{0}\right]=\mathcal{F}\left(\kappa, A_{p}, T_{0}, Q_{m}\right), \quad Q_{0}=\left.\kappa T_{z}\right|_{z=0} .
$$

91 Note that since the model is non-linear an iterative procedure is required for 92 evaluating the operator $\mathcal{K}$ or the function $\mathcal{F}$. We refer to evaluating the operator 
$\mathcal{K}$, for a given heat-flux $Q_{m}$, i.e. solving (2.1), as the direct problem. In contrast solving operator equation (2.2), for a given surface heat-flux $Q_{0}$, is referred to as the inverse problem, see Section 3.

\subsection{Numerical Solution of the Direct Problem}

If the thermal conductivity and the heat production are independent of the temperature, i.e. $\kappa:=\kappa(x, z)$ and $A_{p}:=A_{p}(x, z)$, then solving (2.1) is a standard problem.

By introducing a computational grid $\left(x_{i}, z_{j}\right), i=1, \ldots, N$ and $j=1, \ldots, M$, on the domain $\Omega$, we represent the function $T(x, z)$ by its values at the grid points, i.e. the unknowns are $T=\left(T_{i, j}\right)=\left(T\left(x_{i}, z_{j}\right)\right)$. Similarily the boundary data $T_{0}$ and $Q_{m}$ are represented by vectors, e.g. $Q_{m}=\left(Q_{m}\left(x_{i}\right)\right)$. Thus, having a code for solving (2.1) effectively means that we have have a functional relation

$$
\left[T, Q_{0}\right]=F D M\left(\kappa, A_{p}, T_{0}, Q_{m}\right),
$$

where $T, \kappa$, and $A_{p}$ are matrices, and $T_{0}, Q_{0}$ and $Q_{m}$ are vectors. The above function represents a discrete approximation of the function $\mathcal{F}$, see (2.3), and can be used to approximate the operator $\mathcal{K}$, introduced in $(2.2)$.

In our work we use finite differences [31] to discretize the differential equation. The details of our code are described briefly. At interior points we discretize the governing equation using a symmetric difference approximation,

$$
\left(\kappa T_{x}\right)_{x}\left(x_{i}, z_{j}\right)=\frac{1}{\Delta x^{2}}\left(\kappa_{i-\frac{1}{2}, j} T_{i-1, j}-\left(\kappa_{i-\frac{1}{2}, j}+\kappa_{i+\frac{1}{2}, j}\right) T_{i, j}+\kappa_{i+\frac{1}{2}, j} T_{i+1, j}\right),
$$

where the values of $\kappa(x, z)$ at half-index points are evaluated using linear interpolation. Neumann-type boundary conditions, e.g. $\kappa T_{z}\left(x, L_{z}\right)=Q_{m}(x)$ or $T_{z}(0, z)=0$, are implemented using one-sided finite differences. The finite difference approximation leads to a linear system of equations $A u=b$, where $u$ is a vector that contains the temperature values at the grid points. Having solved for $u$ we can compute the surface heat flux using a one sided difference quotient.

We remark that the linear system $A u=b$ is large and sparse but can be solved effectively using either direct or iterative methods. Also, the matrix $A$ only depends on the thermal conductivity $\kappa$. The righthand side $b$ contains the boundary data, i.e. $T_{0}$ and $Q_{m}$, and also the heat production values $A_{p}$ at interior points. 


\begin{tabular}{|l|c|c|c|}
\hline Layer & $\kappa_{0}\left(\mathrm{~W} / \mathrm{m}^{\circ} \mathrm{C}\right)$ & $\mathrm{c}\left(1 /{ }^{\circ} \mathrm{C}\right)$ & $A_{p}\left(\mathrm{~W} / \mathrm{m}^{3}\right)$ \\
\hline \hline Sediment & 3.0 & 0 & $1.5 \cdot 10^{-6}$ \\
Upper Crust & 3.0 & $1.5 \cdot 10^{-3}$ & $2.0 \cdot 10^{-6}$ \\
Lower Crust & 2.6 & $1.0 \cdot 10^{-4}$ & $0.45 \cdot 10^{-6}$ \\
Mantle & 2.5 & $-2.5 \cdot 10^{-4}$ & $0.02 \cdot 10^{-6}$ \\
\hline
\end{tabular}

Table 1: The parameters used in our model of the thermal conductivity $\kappa$ and heat production $A_{p}$ in the different lithosphere layers, see $[6,11,14]$.

\section{${ }_{122} \quad 2.2 \quad$ A Numerical Example with Artifical Data}

${ }_{123}$ In order to illustrate the geothermal problem we give numerical results for case ${ }_{124}$ where we picked a realistic heat-flux $Q_{m}$ at the base of the model and solved the 125 problem (2.1) using finite differences. Note that this is an artificially generated 126 test problem with a known solution. For the actual application the heat-flux at ${ }_{127}$ the base of the model, i.e. $Q_{m}$, would be unknown. Having a test problem with 128 a known heat flux $Q_{m}$ is convenient for testing purposes.

In our previous paper [9] we used actual measured data at the surface to estimate $Q_{m}$ for the eastern Tibetan margin. In this work we use the same model for the thermal conductivity and the heat production. The thermal conductivity, at crustal conditions, is assumed to vary inversely with temperature [7, 8], i.e.

$$
\kappa=\frac{\kappa_{0}}{1+c T}
$$

where $\kappa_{0}$ is the thermal conductivity at surface conditions and $c$ is an experimentally determined constant. At low temperatures the thermal conductivity generally decreases with increasing temperature, i.e. $c>0$, while in the range $300-500^{\circ} \mathrm{C}$ the conductivity varies very little with temperature. At temperatures above $500^{\circ} \mathrm{C}$ the conductivity instead increases with temperature, i.e. $c<0$ [30]. Further we assume that the heat production, i.e. $A_{p}$ in $(2.1)$, is constant in each lithosphere layer. For discussions of more detailed heat production models see [27, 18]. The specific values used in our simulations are listed in Table 1, see also Figure 1. Note that the heat production is not temperature dependent.

For the numerical simulations, with the above thermal model, we picked a computational grid $\left(x_{i}, z_{j}\right)$, with $N=300$ equidistant grid points in the $x$-direction, and $M=250$ grid points in the $z$-direction. The physical size of the computational domain was where $L_{x}=560 \mathrm{~km}$ and $L_{z}=80 \mathrm{~km}$. At the surface we used a constant temperature $T_{0}=10^{\circ} \mathrm{C}$. The heat-flux $Q_{m}$ is seen in Figure 1 .

Starting from the initial guess $T^{(0)}(x, z)=0$ we used our finite difference code 
to solve (2.1) iteratively,

$$
\left[T^{(k+1)}, Q_{0}^{(k+1)}\right]=F D M\left(\kappa\left(T^{(k)}\right), A_{p}\left(T^{(k)}\right), T_{0}, Q_{m}\right) ;
$$

until the update $\left\|T^{(k+1)}-T^{(k)}\right\|_{F}$ is sufficiently small. At each step we solve a sparse linear system of size $75000 \times 75000$, with 371412 non-zero elements.

The convergence is rather fast, see Figure 1. The final temperature distribution $T(x, z)$, after convergence, is also displayed. We emphasize that the choice of the initial approximation $T^{(0)}(x, z)=0$ is not very good and thus we start with a large error. Still only about five iterations are needed to reach a sufficiently small error.

It is interesting to note that the temperature in the mantle layer varies in the interval $500<T<1150^{\circ} \mathrm{C}$. This means that the thermal conductivity changes significantly, $5.1<\kappa<6.3 \mathrm{~W} / \mathrm{m}^{\circ} \mathrm{C}$ [34], within the mantle layer. Thus a constant value for the thermal conductivity in the whole mantle layer would not give an accurate solution.

\section{The Linear Inverse Problem and Regulariza- tion}

In the previous section we presented the thermal model that is used for our test simulations. Provided that we have access to the heat-flow $Q_{m}$ at the base of the model and the surface temperature $T_{0}$ we can solve (2.1) and obtain the temperature distribution $T(x, z)$. In practice we cannot measure the heatflux $Q_{m}$. Instead we measure both the temperature $T_{0}$ and the heat-flux $Q_{0}$ at surface level; i.e. we have Cauchy data $\left[T_{0}, Q_{0}\right]$ and want to compute the corresponding heat-flux $Q_{m}$. Thus we consider (2.2) as a non-linear operator equation,

$$
\mathcal{K} Q_{m}=Q_{0}, \quad \mathcal{K}:=\mathcal{K}\left(\kappa, A_{p}, T_{0}\right),
$$

that can be solved for the desired heat-flux $Q_{m}$.

In our model the thermal conductivity and heat production are temperature dependent. Even though we are interested in solving the non-linear equation (3.7) we begin by studying the case where the coefficients $\kappa$ and $A_{p}$ are independent of the temperature. We assume that an approximate temperature distribution $T^{(0)}$ is available and use the notation

$$
\kappa:=\kappa(x, z):=\kappa\left(x, z, T^{(0)}\right), \quad \text { and }, \quad A_{p}:=A_{p}(x, z):=A_{p}\left(x, z, T^{(0)}\right),
$$

in the remainder of this section. 

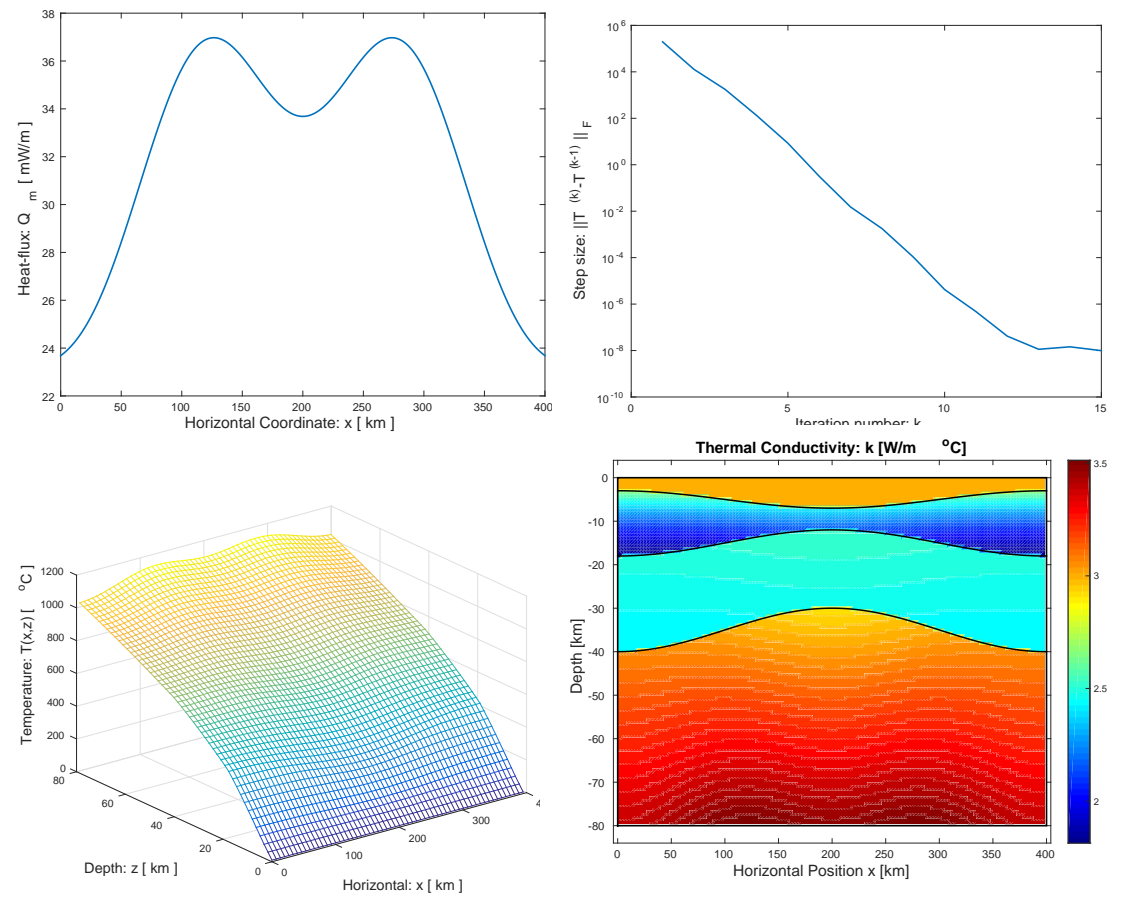

Figure 1: For the test problem we illustrate the heat-flux $Q_{m}$ at the base of the model (top,left), and the convergence history $\left\|T^{(k)}-T^{(k-1)}\right\|_{F}$, measured in the Frobenius norm (top,right). The temperature distribution $T(x, z)$ in the lithosphere (bottom,left) and the thermal conductivity $\kappa(x, z, T)$ (bottom,right) are also displayed.

In the case where the thermal conductivity $\kappa$ and heat production $A_{p}$ are both independent of the temperature we can transform the operator (2.2) into a linear operator. The procedure is as follows: First, find a function $V(x, z)$ that satisfies

${ }_{182}$ (2.1) with the heat-flux $T_{z}\left(L_{z}, x\right)=0$ at the base of the model, i.e.

$$
\left[V, Q_{1}\right]=\mathcal{F}\left(\kappa, A_{p}, T_{0}, 0\right)
$$

Second, set the surface temperature and the heat production to zero, so that ${ }_{184} W$ is defined by

$$
\left[W, Q_{2}\right]=\mathcal{F}\left(\kappa, 0,0, Q_{m}\right)
$$

${ }_{185}$ Then $T=V+W$ and we have $Q_{0}=Q_{1}+Q_{2}$. We conclude that

$$
\widetilde{\mathcal{K}} Q_{m}=Q_{2}=Q_{0}-Q_{1}, \quad \widetilde{\mathcal{K}}=\mathcal{K}(\kappa, 0,0),
$$

${ }_{186}$ and that $\widetilde{\mathcal{K}}$ is a linear operator. 


\subsection{Analysis of the Linear Problem}

In this section we study the linear operator $\widetilde{\mathcal{K}}$ analytically. In order to be able to carry out the analysis we simplify the model and assume that the thermal conductivity $\kappa$ is a constant.

Taking $A_{p}=0, T_{0}=0$ and $\kappa$ a constant, the problem (2.1) transforms into

$$
\begin{cases}T_{x x}+T_{z z}=0, & 0<x<L_{x}, \quad 0<z<L_{z}, \\ T_{x}(0, z)=T_{x}\left(L_{x}, z\right)=0, & 0<z<L_{z}, \\ T(x, 0)=0, & 0<x<L_{x} \\ \kappa T_{z}\left(x, L_{z}\right)=Q_{m}(x), & 0<x<L_{x} .\end{cases}
$$

By separation of variables, we can write the solution as

$$
T(x, z)=\sum_{n=0}^{\infty} a_{n} \cos \left(\frac{n \pi x}{L_{x}}\right)\left(-e^{-\frac{n \pi z}{L_{x}}}+e^{\frac{n \pi z}{L_{x}}}\right) .
$$

The condition $\kappa T_{z}\left(x, L_{z}\right)=Q_{m}(x)$ can also be written as

$$
\kappa T_{z}\left(x, L_{z}\right)=\sum_{n=1}^{\infty} \kappa a_{n} \cos \left(\frac{n \pi x}{L_{x}}\right)\left(\frac{n \pi}{L_{x}} e^{-\frac{n \pi}{L_{x}} L_{z}}+\frac{n \pi}{L_{x}} e^{\frac{n \pi}{L_{x}} L_{z}}\right)=Q_{m}(x),
$$

If we then write $Q_{m}(x)$ as a Fourier cosine series,

$$
Q_{m}(x)=\sum_{n=1}^{\infty} A_{n} \cos \left(\frac{n \pi x}{L_{x}}\right), \text { where } A_{n}=\frac{2}{L_{x}} \int_{0}^{L_{x}} Q_{m}(x) \cos \left(\frac{n \pi x}{L_{x}}\right) d x
$$

and compare the two expressions we find that the constants $a_{n}$ can be written as

$$
a_{n}=\frac{2 \kappa^{-1}}{n \pi\left(e^{-\frac{n \pi L_{z}}{L_{x}}}+e^{\frac{n \pi L_{z}}{L_{x}}}\right)} \int_{0}^{L_{x}} Q_{m}(x) \cos \left(\frac{n \pi x}{L_{x}}\right) d x .
$$

At the earth's surface the heat flux $\kappa T_{z}(x, 0)=Q_{0}$ is then given by

$$
\kappa T_{z}(x, 0)=\sum_{n=1}^{\infty} \kappa \frac{2 n \pi a_{n}}{L_{x}} \cos \left(\frac{n \pi x}{L_{x}}\right)=Q_{0}(x)
$$

substituting for $a_{n}$ and reorganizing the expression gives the result

$$
\int_{0}^{L_{x}} \sum_{n=0}^{\infty} \frac{4}{L_{x}\left(e^{-\frac{n \pi L_{z}}{L_{x}}}+e^{\frac{n \pi L_{z}}{L_{x}}}\right)} \cos \left(\frac{n \pi x^{\prime}}{L_{x}}\right) \cos \left(\frac{n \pi x}{L_{x}}\right) Q_{m}\left(x^{\prime}\right) d x^{\prime}=Q_{0}(x) .
$$
spaces are introduced the operator $\widetilde{\mathcal{K}}$ is compact and its singular system [13] is 
201

$$
\left\{\sigma_{n}, u_{n}, v_{n}\right\}=\left\{\frac{1}{e^{-n \pi \frac{L_{z}}{L_{x}}}+e^{n \pi \frac{L_{z}}{L_{x}}}}, \frac{2}{\sqrt{L_{x}}} \cos \left(\frac{n \pi}{L_{x}} x\right), \frac{2}{\sqrt{L_{x}}} \cos \left(\frac{n \pi}{L_{x}} x\right)\right\} .
$$

The degree of ill-posedness for an operator equation, e.g. (3.10), is defined in

222 where $\lambda>0$ is a regularization parameter. If the matrix $\widetilde{K}$ is explicitly known 223 then the solution can be computed efficiently using the singular value decom224 position [16].

\subsection{Numerical Solution of the Linear Inverse Problem}

After having introduced a computational grid $\left(x_{i}, z_{j}\right)$ the functions $T, \kappa$, and $A_{p}$ are represented by matrices, and $Q_{0}, T_{0}$, and $Q_{m}$ are vectors. The linear operator $\widetilde{\mathcal{K}}$, see $(3.10)$, is approximated by a matrix-relation

$$
\widetilde{K} Q_{m}:=F D M\left(\kappa, 0,0, Q_{m}\right) .
$$

The product of the matrix $\widetilde{K}$ and a vector $Q_{m}$ can be computed by solving a well-posed boundary value problem using our code. Let $\left\{e_{i}\right\}$ be the standard basis on $\mathbb{R}^{N}$. Since the above relation is linear we can compute the matrix $\widetilde{K}$ explicitly by,

$$
\widetilde{K}(:, i)=\widetilde{K} e_{i}=F D M\left(\kappa, 0,0, e_{i}\right), \quad i=1, \ldots, N .
$$

In the remainder of this section we are concerned with solving the ill-conditioned linear system of equations,

$$
\widetilde{K} Q_{m}=\widetilde{Q}_{0},
$$

where the vector $\widetilde{Q}_{0}$ is computed as, see (3.10),

$$
\left[V, Q_{1}\right]=F D M\left(\kappa, A_{p}, T_{0}, 0\right), \quad \text { and } \quad \widetilde{Q}_{0}=Q_{0}-Q_{1} .
$$

The linear system (3.18) is ill-conditioned and regularization is needed[13, 16]. We use Tikhonov regularization[25, 33]; i.e. the regularized solution is computed by minimizing the Tikhonov functional,

$$
\left\|\widetilde{K} Q_{m}^{\lambda}-\widetilde{Q}_{0}\right\|_{2}^{2}+\lambda^{2}\left\|Q_{m}^{\lambda}\right\|_{2}^{2},
$$


The above techniques can be summarized as follows: For the case of temperature independent coefficients the inverse problem (3.7) can be solved using Tikhonov regularization. We obtain a functional relation,

$$
Q_{m}^{\lambda}:=L T\left(\kappa, A_{p}, T_{0}, Q_{0}, \lambda\right)
$$

where, as previously, $\kappa$ and $A_{p}$ are matrices, and $Q_{0}^{\lambda}, T_{0}$, and $Q_{0}$ are vectors, and $\lambda$ is a scalar.

The regularization parameter $\lambda$ controls the weight given to the regularization term, $\lambda^{2}\left\|Q_{m}^{\lambda}\right\|_{2}$, relative to the residual $\left\|\widetilde{K} Q_{m}^{\lambda}-\widetilde{Q}_{0}\right\|_{2}$. If only a little regularization is used, i.e. if $\lambda$ is small, then the noise can be magnified during the computations and destroy the solution. If more regularization is used, i.e. a larger $\lambda$, then the noise is effectively filtered out but the solution may be too smooth and the residual might be large.

The appropriate choice of the parameter $\lambda$ represents a compromise between accuracy, i.e. keeping the residual small, and stability, i.e. preventing the noise from being magnified too much. There are several standard methods for selecting a good regularization parameter for a concrete problem. Two of the most widely used are the Discrepancy principle and the L-curve.

The Discrepancy principle, attributed to Morozov[24], can be used if the noise level in the data is available. The regularization parameter $\lambda$ is then defined as

$$
\lambda:=\sup \left\{\lambda>0 \mid\left\|K Q_{m}^{\lambda}-\widetilde{Q}_{0}^{\delta}\right\| \leq \tau \delta\right\}
$$

where $\delta$ is the noise level, and $\tau \geq 1$ is a suitable constant. This means that the method finds a solution where the residual is of the same order of magnitude as the noise level in the data. The discrepancy principle is known to work well under very general assumptions regarding the ill-posed problem its applied to $[13]$.

The L-curve, originally introduced in [22], see also [17], which is a plot of the residual norm $\left\|K Q_{m}^{\lambda}-\widetilde{Q}_{0}^{\delta}\right\|_{2}$ versus $\left\|Q_{m}^{\lambda}\right\|_{2}$ for different values of $\lambda$, is a convenient graphical tool for analysis of ill-posed problems. The L-curve displays the compromise between the minimization of the two quantities. For discrete ill-posed problems, the L-curve, when plotted in a log-log scale, often has a characteristic L-shaped appearance with a distinct corner separating the vertical and horizontal parts of the curve. Less regularization corresponds to the uppermost part of the curve and more regularization corresponds to the rightmost of the curve. The optimal regularization parameter, that ensures a solution with a good balance between the noise magnification and the approximation error is the value $\lambda$ that corresponds to the corner[16]. 


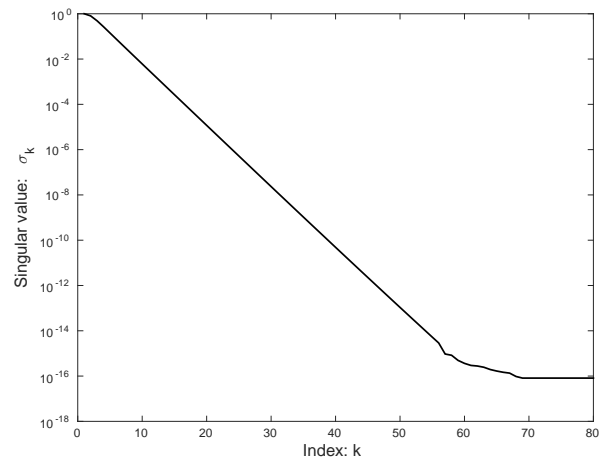

Figure 2: The singular values $\left\{\sigma_{k}\right\}$ for the computed matrix $\widetilde{K}$. The singular values decay exponentially, illustrating that the operator equation $\widetilde{\mathcal{K}} Q_{m}=\widetilde{Q}_{0}$ is severely ill-posed. Only about 60 of the values $\sigma_{k}$ are above the machine precision.

\subsection{A Numerical Example}

In order to illustrate the ill-posedness of the operator $\widetilde{\mathcal{K}}$, and also the effects of and present the results. The test problem is the one presented in Section 2.2, i.e. the grid $\left(x_{i}, z_{j}\right)$ consists of $N=300$ points in the $x$-direction and $M=250$ points in the $z$-direction.

By selecting a vector $Q_{m}$, representing the exact heat flux at the base of the model, and solving the direct problem repeatedly until convergence, we obtain Cauchy data $\left[T_{0}, Q_{0}\right]$, at the surface level, matrices $\kappa$ and $A_{p}$ representing the thermal conductivity and heat production on the grid.

First, using the technique described in Section 3, we compute the matrix $\widetilde{K}$, of size $300 \times 300$, representing the linear operator. Since the matrix is relatively small we can compute its singular value decomposition. The results are displayed in Figure 2. The results clearly demonstrate that the problem is severely ill-posed.

Secondly we add artificially generated noise to the Cauchy data $\left[T_{0}, Q_{0}\right]$. For this test we use normally distributed noise of variance $\epsilon_{1}=5 \cdot 10^{-3}{ }^{\circ} \mathrm{C}$ and $\epsilon_{2}=5 \cdot 10^{-5} \mathrm{~W} / \mathrm{m}^{o} \mathrm{C}$ to obtain the simulated measurements $\left[T_{0}^{\varepsilon_{1}}, Q_{0}^{\varepsilon_{2}}\right]$. The simulated data vectors are illustrated in Figure 3. The noise is of a realistic order of magnitude. In order to find a good value of the regularization parameter we use the $L$-curve, and minimize the Tikhonov functional for a range of parameter values $\lambda$. The method predicts that $\lambda=6.3 \cdot 10^{-3}$ is an appropriate value for the 

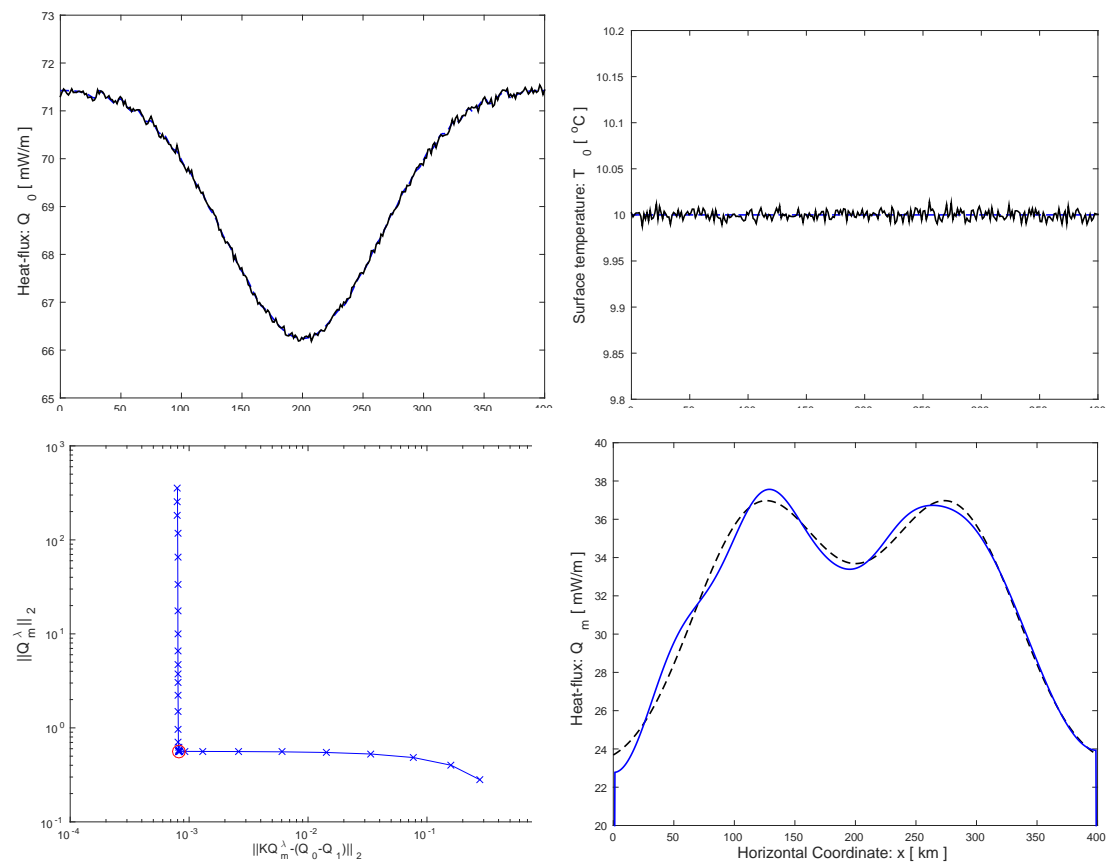

Figure 3: We display the data vectors $Q_{0}^{\epsilon_{2}}$ (top,left) and $T_{0}^{\epsilon_{1}}$ (top,right). In both cases we display the noisy data (black curve) and the exact data (blue curve). The $L$-curve technique is used to identify a suitable value for $\lambda$ (bottom,left). The corner point (red,ring) corresponds to $\lambda=6.3 \cdot 10^{-3}$. The results obtained using $\lambda=6.3 \cdot 10^{-3}$ (bottom,left) are also displayed. The Tikhonov solution $Q_{m}^{\lambda}$ (blue curve) and the exact solution $Q_{m}$ (black dashed curve) are presented.

regularization parameter. Even though the data contains significant amounts of noise the reconstruction of the heat flux at the base of the model is very successful.

In order to illustrate the ill-posedness of the problem we also solve the equation using less regularization. The solution $Q_{m}^{\lambda}$ computed using $\lambda=7 \cdot 10^{-4}$ is displayed in Figure 4. Clearly the solution is highly oscillating as a result of noise magnification during the computations. Also we present a solution computed using $\lambda=9 \cdot 10^{-2}$. In this case too much regularization results in a too smooth solution and features from the exact solution are not reconstructed accurately. 

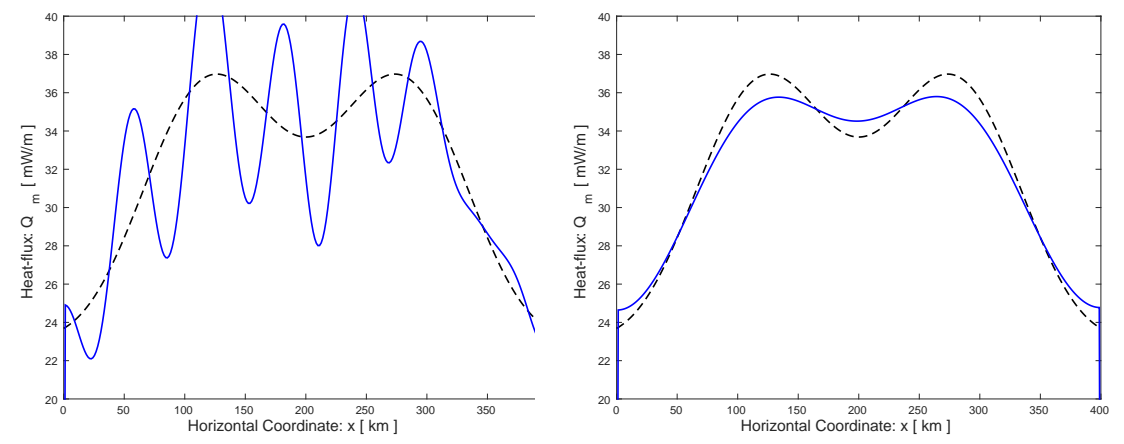

Figure 4: We display the Tikhonov solutions $Q_{m}^{\lambda}$, using $\lambda=7 \cdot 10^{-4}$ (left, blue curve) and $\lambda=9 \cdot 10^{-2}$ (right, blue curve). In the first case the not enough regularization has been applied and in the second case we have too much regularization. The exact solution $Q_{m}$ (black dashed curve) is also presented.

\section{The Non-linear Problem}

We recall that our goal is to solve the non-linear case, where both the thermal conductivity $\kappa$ and the heat production $A_{p}$ depend on the temperature. Our idea is to solve a sequence of linear problems using Tikhonov regularization. Let $T^{(0)}$ be an approximate temperature distribution. The algorithm is as follows: First solve the linearized problem using Tikhonov regularization to obtain

$$
Q_{m}^{(k+1), \lambda}:=\operatorname{LT}\left(\kappa\left(T^{(k)}\right), A_{p}\left(T^{(k)}\right), T_{0}, Q_{0}, \lambda\right) .
$$

The new approximate heat-flux $Q_{m}^{(k+1), \lambda}$, at the base of the model, is then used to compute the next temperature distribution

$$
T^{(k+1)}:=F D M\left(\kappa\left(T^{(k)}\right), A_{p}\left(T^{(k)}\right), T_{0}, Q_{m}^{(k+1), \lambda}\right) .
$$

The process is repeated until the update $\left\|T^{(k+1)}-T^{(k)}\right\|_{F}$ is sufficiently small.

The algorithm works well, provided that a sufficiently large regularization parameter $\lambda$ is used, but is computationally demanding. At each step we need to form a linear system of equations $A u=b$, see Section 2.1. For relatively small grid sizes, i.e. $2 D$ calculations, it is feasible to solve the linear system using Gaussian elimination. If the matrix $\widetilde{K}$, see $(3.17)$, is desired then we make use of the observation that the matrix $A$ only depends on the thermal conductivity $\kappa\left(T^{(k)}\right)$. Thus we can compute the $L U$ decomposition of the sparse matrix $A$ and reuse it when evaluating $\widetilde{K} e_{i}$, for all the basis vectors $e_{i}$. After having computed the matrix $\widetilde{K}$ explicitly we can easily find the regularized solution of the inverse problem by solving the least squares problem (3.20). 


\subsection{Modified Normal Equations and an Iterative Method}

For large grid sizes, e.g. $3 D$ calculations, it is necessary to avoid forming $\widetilde{K}$, see (3.17), explicitly. Instead we note that the minimization problem (3.20) is equivalent to solving the modified normal equations,

$$
\left(\widetilde{K}^{T} \widetilde{K}+\lambda^{2} I\right) Q_{m}^{\lambda}=\widetilde{K}^{T} \widetilde{Q}_{0} .
$$

Instead of forming the matrix $\widetilde{K}$ explicitly we solve the normal equations using the conjugate gradient method[28]. The matrix-vector product $\widetilde{K} Q_{m}$ can be evaluated by solving the direct problem using finite differences, i.e.

$$
\widetilde{K} Q_{m}:=F D M\left(\kappa, 0,0, Q_{m}\right) .
$$

The core of the finite difference code is a linear system of equations $A u=b$, where $u_{k}=T\left(x_{i}, z_{j}\right), k=(i-1) n+j$. The right hand side $b$ is zero except for a block that contains the vector $Q_{m}$. In matrix form this can be written as

$$
b=W_{2} Q_{m}, \quad W_{2} \in \mathbb{R}^{N M \times N},
$$

where $W_{2}$ is a matrix that places the values in $Q_{m}$ at the correct locations in the vector $b$. After having computed the temperatures $T_{i j}$ at the grid points a finite difference formula is used to obtain the heat-flux at the surface level. This can also be represented in matrix form as

$$
\widetilde{Q}_{0}=W_{1} u, \quad W_{1} \in \mathbb{R}^{N \times N M},
$$

where $W_{1}$ is a matrix that computes a finite difference approximation of the surface heat flux given the temperature values stored in the vector $u$. The matrix $\widetilde{K}$ can be written in factorized form as,

$$
\widetilde{Q}_{0}=W_{1} u=W_{1} A^{-1} b=W_{1} A^{-1} W_{2} Q_{m}=\widetilde{K} Q_{m},
$$

and its transpose can be written as,

$$
\widetilde{K}^{T}=W_{2}^{T}\left(A^{T}\right)^{-1} W_{1}^{T} .
$$

In order to evaluate a matrix-vector product $\widetilde{K}^{T} Q$ we need to solve a system of equations involving the matrix $A^{T}$. Our finite difference code is written so it can compute matrix-vector products involving both $\widetilde{K}$ and $\widetilde{K}^{T}$. In order to evaluate the product,

$$
\left(\widetilde{K}^{T} \widetilde{K}+\lambda^{2} I\right) Q_{m}
$$

we need to form the matrix $A$ once and we need to solve two linear systems of equations (one with $A$ and one with $A^{T}$ ). Note that if the $L U$ decomposition of $A$ is computed then the relation $A^{T} P^{T}=U^{T} L^{T}$ can be used to solve the system involving $A^{T}$ efficiently. If the matrix $A$ is too large, e.g. the $3 D$ case, then instead we use an iterative method, e.g. CG or GMRES[28]. 
Thus in the implementation of (4.23) the minimization problem (3.20) is solved by finding the solution $Q^{(k+1), \lambda}$ of the linear system of equations,

$$
\left(\left(\widetilde{K}^{(k)}\right)^{T} \widetilde{K}^{(k)}+\lambda^{2} I\right) Q_{m}^{(k+1), \lambda}=\left(\widetilde{K}^{(k)}\right)^{T} \widetilde{Q}_{0},
$$

where $\widetilde{K}^{(k)}$ is the matrix defined by the relation (4.26) above; with $\kappa:=\kappa\left(T^{(k)}\right)$. The previous solution $Q_{m}^{(k), \lambda}$ can be used as an initial guess to start the CG iterations.

\subsection{Numerical Tests for the Non-Linear Problem}

Here we present the results from the numerical testing of the methods. For all tests we use the numerically constructed example from Section 2.2. The grid $\left(x_{i}, z_{j}\right)$ consists of $N=300$ points in the $x$-direction and $M=250$ points in the $z$-direction. For the tests a vector $Q_{m}$, representing the exact heat-flux at the base of the model, was selected and the direct problem solved repeatedly until convergence. In all cases the direct problems were solved with sufficient accuracy that the generated Cauchy data $\left[T_{0}, Q_{0}\right]$ can be considered exact.

To the generated Cauchy data we add normally distributed noise of variance $\epsilon_{1}$ and $\epsilon_{2}$ to obtain the simulated measurements $\left[T_{0}^{\varepsilon_{1}}, Q_{0}^{\varepsilon_{2}}\right]$. The vectors $T_{0}$ and $Q_{0}$ are of different orders of magnitude so it is important to use different noise levels for the temperature and heat-flux vectors respectively. For our tests we used the noise levels $\epsilon_{1}=5 \cdot 10^{-3}{ }^{\circ} \mathrm{C}$ and $\epsilon_{2}=5 \cdot 10^{-5} \mathrm{~W} / \mathrm{m}^{\circ} \mathrm{C}$. The simulated data vectors are illustrated in Figure 5 . The noise levels are realistic. The same data vectors and noise are are used for all tests presented in the text below.

The purpose of the tests is to illustrate how the algorithm for solving the non-
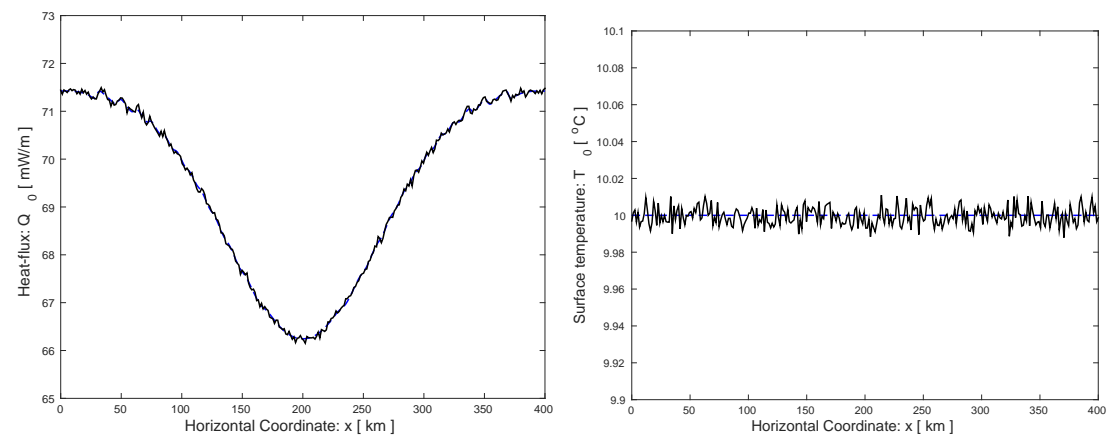

Figure 5: The simulated Cauchy data at the surface level. We display the heatflux $Q_{0}$ (left) and the temperature $T_{0}$. In both cases we display both the exact function (blue,dashed curve) and the noisy function (black, solid curve). 

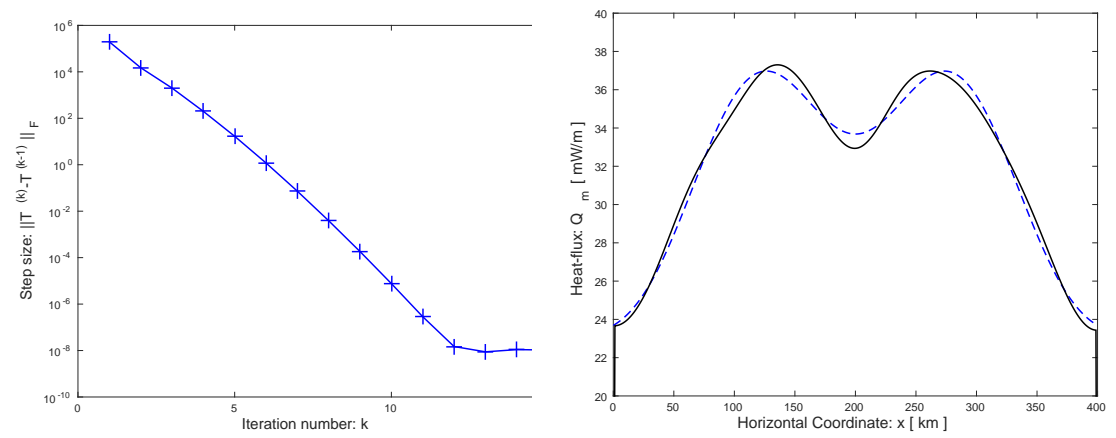

Figure 6: Results for non-linear Tikhonov regularization. We display the heatflux $Q_{m}$ at the base of the model (right,dashed blue curve) the approximation $Q_{m}^{(10), \lambda}$ (right,black curve), and the convergence history $\left\|T^{(k), \lambda}-T^{(k-1), \lambda}\right\|_{F}$, measured in the Frobenius norm (left).

linear problem, i.e. the steps (4.24) and 4.24), works in practice. For the Tikhonov regularization to work well a good regularization parameter is needed. We suggest that the $L$-curve is used and in our tests we use the value $\lambda=$ $6.3 \cdot 10^{-3}$ that was obtained by the $L$-curve for the constant coefficient case, see Section 3.2 .

For the first test we use the simplest implementation of Tikhonov regularization, i.e. in each step the matrix $\widetilde{K}^{(k)}$, defined by the relation (3.18), is computed explicitly and the minimization problem (3.20) is solved by computing the SVD of the matrix. It is also assumed that the linear systems of equations that arise due to the finite difference discretizations are solved using a sparse $L U$ decomposition.

The results are presented in Figure 6. The convergence is rapid and only 3 iterations is enough to reach a level where the first decimal digits of the temperatures $T^{(k)}$ stops changing. The large initial error is due to the poor starting guess $T^{(0)}=0$.

We remark that this is a very inefficient approach. At each step of the algorithm we need to solve two different types of finite difference equations. First, the functional relation (3.19) is implemented by first forming the matrix in the finite difference equations and computing its $L U$ decomposition. Second, we need to form the finite difference equations defined by the relation (3.16), compute its $L U$ decomposition and solve $2 N$ triangular linear systems to obtain the matrix $\widetilde{K}^{(k)}$. Minimizing the small Tikhonov functional, e.g. (3.20), is cheap by comparison and the cost can be neglected.

In our second test we solve the same problem again using the iterative implemen- 

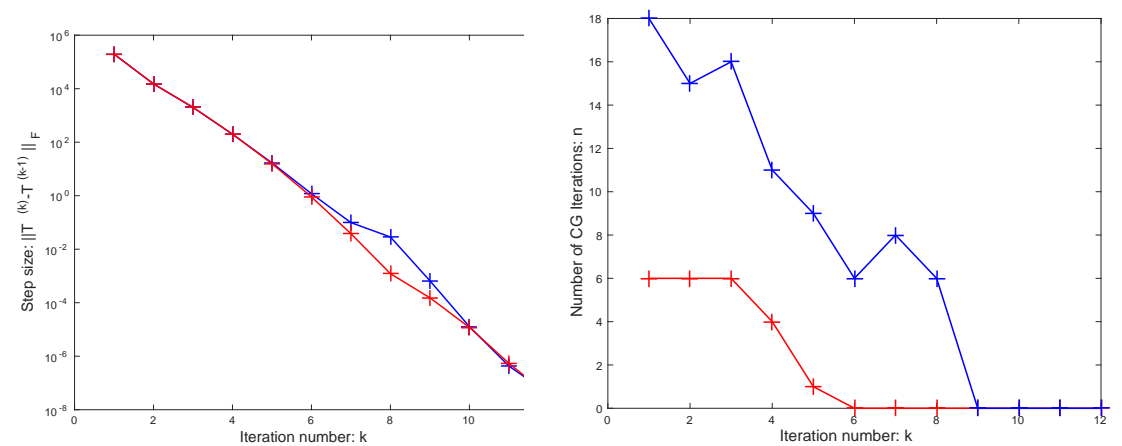

Figure 7: Results obtained using the iterative implementation of Tikhonov regularization. We display the convergence history $\left\|T^{(k), \lambda}-T^{(k-1), \lambda}\right\|_{F}$, measured in the Frobenius norm (left) and also the number of CG iterations needed to reach the stopping criteria at each step of the algorithm (right). The stopping criteria for the CG iterations were set at either $10^{-9}$ (blue curves) and $10^{-5}$ (red curves).

tation of Tikhonov regularization, see Section 4.1. The starting guess $T^{(0)}=0$ was used and the stopping criteria for the CG iterations was a relative residual of magnitude less than $10^{-9}$. The results are illustrated in Figure 7 . Note that after iteration 8 the solution from the previous step already satisfies the stopping criteria and the algorithm can be said to have converged to desired precision, also as the quality of the solution improves fewer CG iterations are needed. The obtained solution $Q_{m}^{(8), \lambda}$ is presented in Figure 8. The quality of the obtained solution is the same as the one seen previously in Figure 6 . Note that even $Q^{(k), \lambda}$ has converged the temperature $T^{(k), \lambda}$ can still change due to the fact that the problem is non-linear and $\kappa\left(T^{(k), \lambda}\right)$ is recomputed in each step.

The computational work for the second test is as follows. For each step of the algorithm we need to form two linear systems corresponding to (3.19) and (3.16). The first system is solved once and the second needs to be solved twice during each step in the CG algorithm. The amount of work required is significantly less than for the first test above.

In order to further reduce the amount of computational work we stop the CG iterations when the relative residual is less than $10^{-5}$. This means the linear systems are solved less accurately. The results are also displayed in Figure 7. Here it is enough to perform 5 iterations to reach convergence. A total 10 systems of finite difference equations needs to be formed, and 58 linear system solves are needed. The obtained solution $Q_{m}^{(5), \lambda}$, after 5 steps of the algorithm, is displayed in Figure 8. 

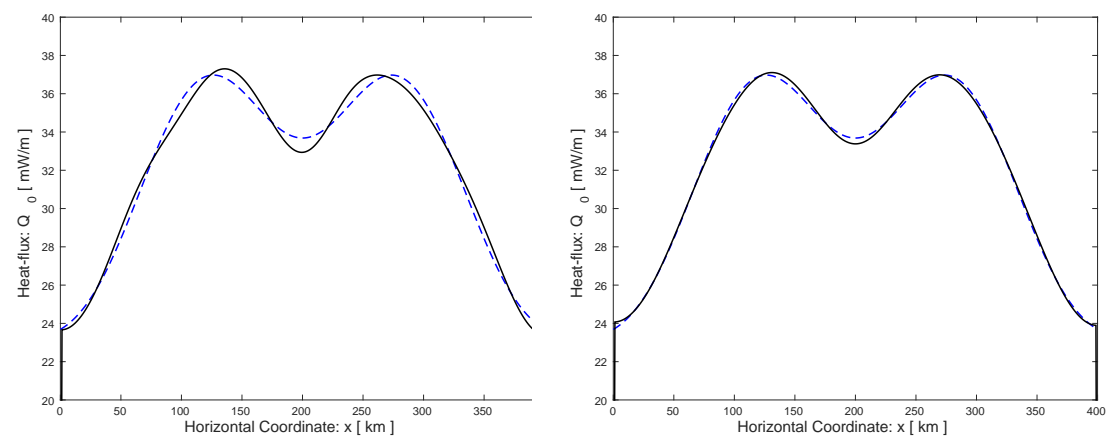

Figure 8: Results for the iterative implementation of Tikhonov regularization. We display the heat-flux $Q_{m}$ at the base of the model (dashed blue curves) and the computed approximations $Q_{m}^{(8), \lambda}$ (left,black curve), for the case then the stopping in the $\mathrm{CG}$ algorithm criteria was set to $10^{-9}$ and also the solution $Q_{m}^{(5), \lambda}$ (right, black curve) for the case when the stopping criteria were $10^{-5}$. The two solutions are of equally good quality.

\section{Concluding Remarks}

In this paper we have presented the inverse geothermal problem. The application is important since many geophysical process are influenced by the temperature. The physical properties of rocks depend strongly on the temperature and the problem is non-linear.

We have demonstrated that the problem is severely ill-posed and regularization is needed. One of the most popular regularization techniques is Tikhonovs method. The method requires that we rewrite the geothermal problem as an operator equation, and minimize a related least squares problem. The tests in the paper demonstrates that this approach works well. Though the results may be sensitive with respect to the thermal model used the influence from random measurement errors is relatively small and the computations are numerically stable.

Our implementation is based on having a finite difference solver for a related, well-posed, direct problem. We show how to use software for the direct problem when implementing Tikhonov regularization for the inverse problem. Our algorithm is iterative and the conjugate gradient method is used for solving the normal equations that appear due to the Tihonov regularization. The method is efficient and solving the inverse problem requires only about an order of magnitude additional work compared to solving only the direct problem. It is worth noting that instead starting from a finite element solver for the direct problem would work equally well. 
In our work we consider only $2 D$ models however the algorithm can be expected to work well for $3 D$ calculations as well. As long as the corresponding direct problem can be solved we can also solve the inverse problem. Calculations on a realistic full $3 D$ model is something we intend to do in the future.

\section{References}

[1] I.M. Artemieva and W.D. Mooney. Thermal thickness and evolution of precambrian lithosphere: A global study. Journal of Geophysical Research, 106:16387-16414, 2001.

[2] F. Berntsson and L. Eldén. Numerical solution of a Cauchy problem for the laplace equation. Inverse Problems, 17(4):839-853, 2001.

[3] J. Braun. Hot blanket in earths deep crust. Nature, (458):292-29, 2009.

[4] G. Bruckner, S. Handrock-Meyer, and H. Langmach. An inverse problem from 2d ground-water modelling. Inverse Problems, 14(4):835-851, 1998.

[5] V. Cermak and L. Bodri. Two-dimensional temperature modelling along five east-european geotraverses. Journal of Geodynamics, 5(2):133 - 163, 1986.

[6] V. Cermak, L. Bodri, R. Schulz, and B. Tanner. Crustal temperatures along the central segment of the european geotraverse. Tectonophysics, 195(24):241 - 251, 1991.

[7] V. Cermak and L. Rybach. Geophysics - Physical Properties of Rocks, chapter Thermal Conductivity and Specific Heat of Minerals and Rocks, page 305343. Springer, 1982.

[8] D. S. Chapman. Thermal gradients in the continental crust. Geological Society, London, Special Publications, 24(1):63-70, 1986.

[9] Lin Chen, Fredrik Berntsson, Zhongjie Zhang, Peng Wang, Jing Wu, and Tao Xu. Seismically constrained thermo-rheological structure of the eastern tibetan margin: Implication for lithospheric delamination. Tectonophysics, 627(0):122-134, 2014.

[10] Wang-Ping Chen, Chun-Quan Yu, Tai-Lin Tseng, Zhaohui Yang, Chi yuen Wang, Jieyuan Ning, and Tiffany Leonard. Moho, seismogenesis, and rheology of the lithosphere. Tectonophysics, 609(0):491-503, 2013.

[11] C. Clauser. Heat transport processes in the earths crust. Survey in Geophysics, 30:163-191, 2009. 
[12] Christoph Clauser, Peter Giese, Ernst Huenges, Thomas Kohl, Holger Lehmann, Ladislaus Rybach, Jan afanda, Helmut Wilhelm, Karla Windloff, and Gustav Zoth. The thermal regime of the crystalline continental crust: Implications from the ktb. Journal of Geophysical Research: Solid Earth, 102(B8):18417-18441, 1997.

[13] H. Engl, M. Hanke, and A. Neubauer. Regularization of Inverse Problems. Kluwer Academic Publishers, Dordrecht, the Netherlands, 1996.

[14] Kevin P. Furlong and David S. Chapman. Heat flow, heat generation, and the thermal state of the lithosphere. Annual Review of Earth and Planetary Sciences, 41(1):385-410, 2013.

[15] J. Hadamard. Lectures on Cauchy's problem in linear partial differential equations. Dover Publications, New York, 1953.

[16] P. C. Hansen. Rank-Deficient and Discrete Ill-Posed Problems. Numerical Aspects of Linear Inversion. Society for Industrial and Applied Mathematics, Philadelphia, 1997.

[17] P. C. Hansen and D. P. O'Leary. The use of the L-Curve in the regularization of ill-posed problems. SIAM J. Sci. Comput., 14:1487-1503, 1993.

[18] D. Hasterok and D.S. Chapman. Heat production and geotherms for the continental lithosphere. Earth and Planetary Science Letters, 307(12):59 $70,2011$.

[19] Lin Chen; Taras Gerya; Zhongjie Zhang; Guizhi Zhu; Thibault Duretz; Wolfgang R. Jacoby. Numerical modeling of eastern tibetan-type margin: Influences of surface processes, lithospheric structure and crustal rheology. Gondwana Research, 24(3-4):1091-1107, 2013.

[20] Claude Jaupart and Jean-Claude Mareschal. Heat generation and transport in the Earth. Cambridge university press, 2010.

[21] A.A. Kremenetsky, S.Yu. Milanovsky, and L.N. Ovchinnikov. A heat generation model for continental crust based on deep drilling in the baltic shield. Tectonophysics, 159(34):231 - 246, 1989.

[22] C. L. Lawson and R. J. Hanson. Solving Least Squares Problems. PrenticeHall, Englewood Cliffs, NJ, 1974.

[23] JC Mareschal and C Jaupart. Variations of surface heat flow and lithospheric thermal structure beneath the north american craton. Earth and Planetary Science Letters, 223(1):65-77, 2004.

[24] V. A. Morozov. On the solution of functional equations by the method of regularization. Soviet. Math. Dokl., 7:414-417, 1966.

[25] D.L. Phillips. A technique for the numerical solution of certain integral equations of the first kind. J. Assoc. Comput., pages 84-97, 1962. 
[26] Roberta L Rudnick, William F McDonough, and Richard J O'Connell. Thermal structure, thickness and composition of continental lithosphere. Chemical Geology, 145(3):395-411, 1998.

[27] L Rybach and G Buntebartw. The variation of heat generation, density and seismic velocity with rock type in the continental lithosphere. Tectonophysics, 103:335-344, 1984.

[28] Y Saad. Iterative Methods for Sparse Linear Systems. International Thomson Publishing, 1996.

[29] J. Safanda and A. Janackova. Calculation of temperature distribution in two-dimensional geothermal profile. Studia Geophysica et Geodaetica, 29(2):191-207, 1985.

[30] John F. Schatz and Gene Simmons. Thermal conductivity of earth materials at high temperatures. Journal of Geophysical Research, 77(35):69666983, 1972 .

[31] G. D. Smith. Numerical Solution of Partial Differential Equations. Oxford University Press, Oxford, third edition, 1985.

[32] D. Stromeyer. Downward continuation of heat flow data by means of the least squares method. Tectonophysics, 103(14):55 - 66, 1984.

[33] A. N. Tikhonov. Solutions of incorrectly formulated problems and the regularization method. Soviet Math. Dokl., 4:1035-1038, 1963.

[34] Yousheng Xu, Thomas J Shankland, Sven Linhardt, David C Rubie, Falko Langenhorst, and Kurt Klasinski. Thermal diffusivity and conductivity of olivine, wadsleyite and ringwoodite to $20\{\mathrm{GPa}\}$ and $1373 \mathrm{k}$. Physics of the Earth and Planetary Interiors, 143144:321 - 336, 2004.

[35] M. Zhdanov. Inverse Theory and Applications in Geophysics. Elsevier Science, 2nd edition, 2015. 